\title{
Cytological factors relating to posterior capsule opacification following cataract surgery
}

\author{
T J C JACOB,' R C HUMPHRY, ${ }^{2}$ E G DAVIES, ${ }^{2}$ AND \\ G M THOMPSON ${ }^{2}$
}

From the 'Department of Pharmacology St George's Hospital Medical School, and the 'Department of Ophthalmology, St George's Hospital, London SW17 ORE

SUMMARY Simulated extracapsular cataract extractions on cadaver eyes were performed which demonstrated that the cells of the anterior capsule remain largely intact and that only a small amount of cortical lens matter remains postoperatively. Human lens epithelial cells from normal and cataractous lenses were grown in culture. There was no appreciable difference in growth rate between cells from normal and those from cataractous lenses or between equatorial and central capsule cells. The cells grew from the cut edges of the capsule, suggesting that release from contact inhibition is an important factor in stimulating proliferation. The daughter cells became increasingly abnormal and metaplastic in successive generations, but there was no evidence of differentiation into fibroblasts within the 35-day culture period, suggesting that a retinal growth factor may be involved with the fibrosis occurring in opacification of the posterior capsule. A small anterior capsulotomy will release fewer cells from contact inhibition and hence reduce cell proliferation after extracapsular cataract extraction.

The resurgence of the extracapsular technique of cataract extraction has been accompanied by a corresponding increase in the prevalence of postoperative posterior capsule opacification. ' The effectiveness of a cataract extraction, with or without an intraocular lens, may be considerably reduced when severe opacification occurs. Although the capsule membrane may be ruptured by YAG laser capsulotomy,$^{23}$ this is a second procedure that is associated with further complications and may be difficult in elderly patients. If a greater understanding of the mechanisms underlying opacification of the posterior capsule could lead to its decreased incidence, there would be considerable clinical benefit.

Our study was in two parts: firstly, we obtained enucleated human eyes and performed an extracapsular lens extraction under operating theatre conditions. These eyes were examined histologically in order to determine which elements of the lens remained after an, apparently, complete extracapsular lens extraction.

Correspondence to R C Humphry, FRCS, West of England Eye Infirmary, Magdalen Street, Exeter.
Secondly we obtained normal and cataractous human lenses and examined the growth of the epithelial cells in cell culture in order to determine the best type of capsulotomy in extracapsular surgery. In particular: (a) The pattern of growth from capsule edges; whether removal of intercell contact inhibition is a factor in stimulating epithelial proliferation. (b) Whether cells from different parts of the capsule grow at different rates - in other words, does the site of the capsulotomy influence epithelial cell proliferation? (c) Whether epithelial cells from cataractous lenses grow at a different rate from those of normal lenses.

\section{Material and methods}

SIMULATED EXTRACAPSULAR CATARACT EXTRACTION

An extracapsular lens extraction was performed under standard theatre conditions on fresh cadaver eyes. This involved a corneal section, anterior capsulotomy, nucleus expression and lens matter aspiration with a hand held irrigation/aspiration coaxial cannula (0.3 mm port). Surgery was carried out under an 
operating microscope, and as complete a removal as possible was achieved, with Hartmann's solution for irrigation.

The eyes were then fixed in $10 \%$ formalin and washed in methyl alcohol. The specimens were cooled in isopentane and nitrogen to facilitate sectioning the globe in a coronal place posterior to the lens capsule. The anterior segment was embedded in paraffin wax and stained with haematoxylin and eosin, and sectioned for histological examination.

\section{TISSUE CULTURE}

Normal lens epithelial tissue was obtained from human donor eyes used for corneal graft surgery within 36 hours of the death of the donor. The eye was bisected equatorially behind the ora serrata and the lens removed by cutting the suspensory ligaments. Cataractous lenses were obtained from patients at surgery by an intracapsular technique using forceps. The lens was then pinned, anterior surface down, to a Sylgard pad. After a circular incision had been made round the equator the posterior capsule, nucleus, and cortical lens matter were removed. The remaining anterior capsule with adherent epithelium was cut into rectangular strips such that each strip spanned the diameter of the lens capsule. Hence the central and equatorial cells were cultured.

The epithelial strips were laid flat on collagen coated Petri dishes and incubated in medium E199 + $50 \%$ fetal calf serum (FCS), penicillin/streptomycin (50 units $/ \mathrm{ml}, 50 \mathrm{mg} / \mathrm{l}$ respectively) at $37^{\circ} \mathrm{C}$ (in a humidified atmosphere containing 5\% $\mathrm{CO}_{2}$ ). The serum concentration in the medium, originally $50 \%$, was reduced to $36 \%$ on day $10,25 \%$ on day 13 , and $10 \%$ on day 16 . Thereafter the medium ( $+10 \%$ FCS $)$ was changed every three days.

CELL COUNTING

The outgrowth of cells was examined either by direct observation under phase contrast optics $(\times 4$, Nikon inverted microscope) or after fixation (ethanol:acetic acid), staining (Giemsa), and mounting (Aqualite).

Cellular outgrowth was determined by counting the number of cells along an axis perpendicular to the cut edge of the rectangular explant: (1) by means of photographic enlargements of fixed/stained cultures, or (2) directly on growing cultures under phase contrast optics.

To measure the outgrowth from specific regions of the explant after 35 days the fixed/stained cultures were examined under the microscope $(\times 26)$; with a calibrated eyepiece graticule the extent of the outgrowth along three axes perpendicular to the explant edge was measured at the equatorial and central regions respectively, and these values were averaged. The results could be expressed in terms of length, since variations in cell size were uniform within each culture, and each culture acted as its own control. This was not possible for comparisons of outgrowth between normal and cataractous lenses, since cell size increases with age of the culture. Thus, to avoid the possible inaccuracies that this size variation might introduce to measurements of outgrowth dimensions between cultures, it was decided to count individual cells along four axes perpendicular to the cut edge of the explant. Counts made on fixed/stained material, where much more detailed study was possible, acted as a check on the accuracy of the method, and all counts made by the method outlined above lay within one standard deviation of this more exhaustive method. Fig. 2 includes both sets of measurements.

\section{AREA DETERMINATION}

The area of cellular outgrowth was determined after 35 days of culture. The cultures were fixed, stained, and mounted as described above and placed over a grid (1 mm squares) and viewed under a dissecting microscope $(\times 20)$. The total area (outgrowth plus explant) was determined by counting the number of squares covered and subtracting the area of the explant. The average cell diameter was determined by counting the number of cells along measured axes from the edge of the explant to the periphery of the outgrowth. With these two parameters it was possible to calculate the number of cells in the outgrowth. This value is an approximation and was not used as a criterion in experimental tests.

From the total number of cells produced before proliferation ceased and the number of parent cells (that is, the number of cells at the edge of the explant) the population doubling level (PDL) was calculated;

$$
\begin{aligned}
& P D L=\log _{10}\left(N_{1} / N_{0}\right) / \log _{10}{ }^{2} \ldots(1) \\
& \text { where } N_{1}=\text { total number of cells, } \\
& N_{0}=\text { number of parent cells. }
\end{aligned}
$$

\section{Results}

After 35 days the cultures were fixed, stained, and mounted and the total area of cellular outgrowth was determined as described in 'Material and methods.' The average area of outgrowth for nine capsular/ epithelial strips after 35 days in culture was 85.4 (SE 8.4$) \mathrm{mm}^{2}(\mathrm{n}=9)$. The cell diameter varied considerably from the edge of the explant to the periphery of the outgrowth, and this was a feature of all cultures. In a minority of cultures a limited degree of cell elongation was observed. Near the explant the cell diameter ranged from 30 to $50 \mu \mathrm{m}$, while some cells in the outer regions had diameters in excess of $100 \mu \mathrm{m}$. This compares with a diameter of $12 \mu \mathrm{m}$ for parent cells on the explant. From the area, $\pi \mathrm{r},{ }^{2}$ where $\mathrm{r}=21.5 \mu \mathrm{m}$ (calculated as described in 'Material and methods'), and the total area of cellular 


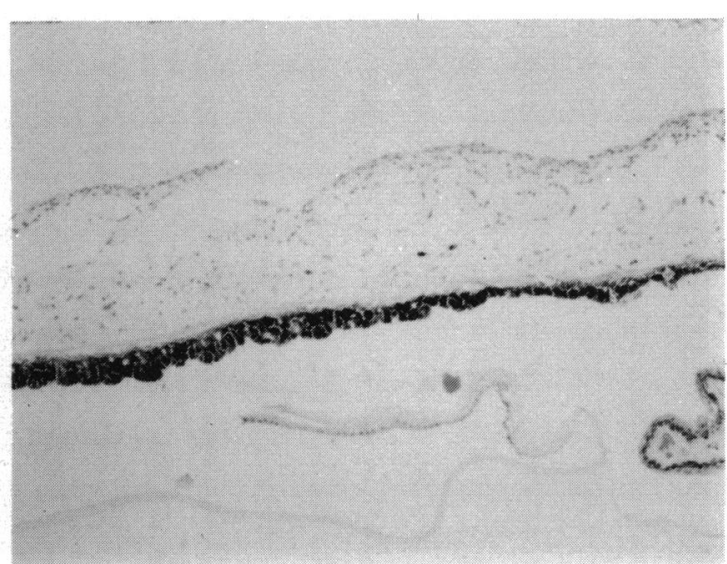

Fig. 1A

Fig. 1A Cross section of the human lens after simulated extracapsular lens extraction. While most of the soft lens matter is removed by irrigation, epithelial cells remain attached to the anterior and equatorial regions of the capsule.

outgrowth it is possible to make an estimate of the number of new cells produced before the cessation of proliferation. For nine normal lens epithelial explants the average was 45000 . The population doubling level (PDL) can be calculated (equation 1) if the initial number of cells is known. The average area of the explants was 5.7 (SE 0.6) $\mathrm{mm}^{2}$, which gives a value of 600-800 edge cells. On the assumption that these are the only cells available for proliferation, then from equation (1) the PDL achieved by these cultures of human lens epithelial cells was 6 .

\section{EXTRACAPSULAR CATARACT EXTRACTION IN VITRO}

Histological study of the sections of the eyes showed that the remaining capsular bag was largely free of cortical lens matter (Fig. 1A), although small quantities were left in the equatorial regions (Fig. 1B). It was notable that in every case the epithelial cells remained intact and attached to the anterior and equatorial lens capsule. Normal irrigation/aspiration with Hartmann's solution did not remove these cells, which remained in their normal configuration on the anterior capsule remnants. There were no epithelial cells posterior to the equator.

CULTURED LENS CAPSULAR/EPITHELIAL STRIPS After 3-5 days in culture cellular outgrowth from the explanted tissue was visible. This outgrowth continued as a monolayer for approximately 30 days and then stopped (Fig. 2), by which time the new cells extended about $5 \mathrm{~mm}$ from the edge of the explant.

In Fig. 2 the growth kinetics of explants from seven cataractous lenses and five normal lenses (six explants

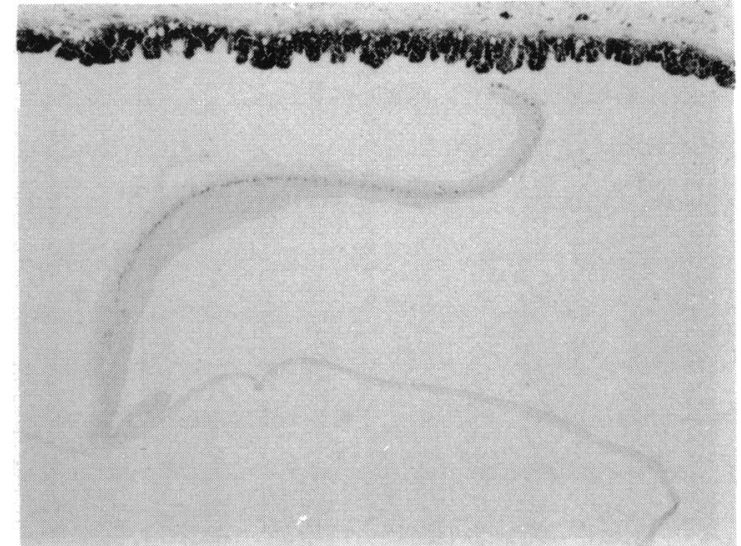

Fig. 1B

Fig. 1B Enlargement of the equatorial region of the lens illustrating some residual cortical lens matter. The anterior and equatorial epithelial cells remain intact.

from each lens) are compared. The data are presented as the average number of cells along an axis perpendicular to the cut edge of the explant (see 'Cell counting' under 'Material and methods'), as a function of time in culture. After a lag phase of about 2-3

CELL GROWTH FROM HUMAN LENS EPITHELIAL EXPLANTS

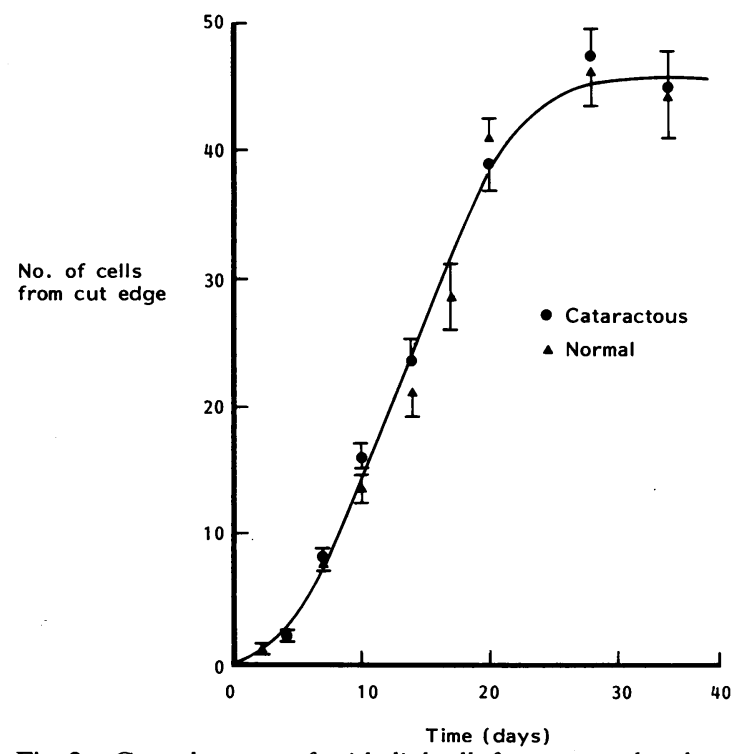

Fig. 2 Growth curves of epithelial cells from normal and cataractous lenses. The rate of outgrowth (y axis) is expressed as the number of cells from the cut edge of the capsularl epithelial strip. Each point represents the mean $\pm S E$ (bars) of explants from seven cataractous and five normal lenses. The plateau value of cellular proliferation represents six population doublings (see text). 


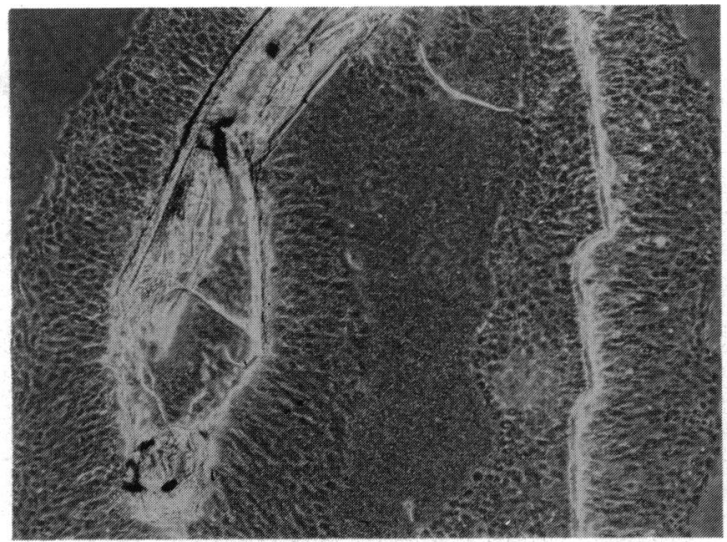

Fig. 3A: Equatorial region of lens capsule.

Fig. 3 Anteroposterior phase contrast micrographs of lens capsule strips after seven days in tissue culture. Daughter cells are growing both away from the cut edges on to the collagen substrate and also over the anterior surface of the capsule.

days the cells divided exponentially, reaching a plateau after about 28 days. No difference in growth characteristics was observed between explants from cataractous and normal lenses. At this stage no attempt was made to differentiate between types of cataract. Both cortical and nuclear as well as mixed cataractous types were used.

In neither normal nor cataractous explants was any difference in outgrowth between different regions along the strip of explant observed; central epithelial cells proliferated at the same rate as epithelial cells from the peripheral, germinative region (Fig. $3 \mathrm{~A}$ and B), after 35 days the outgrowth extended 4.0 (SE 0.4) $\mathrm{mm}$ from the peripheral region and 4.4 (SE 0.3$) \mathrm{mm}$ from the central region $(n=9)$.

The cells grew from the cut edge of the capsular/ epithelial strip, not only on to the surface of the collagen substrate but also around and on to the anterior surface of the capsule (Fig. 3B).

\section{Discussion}

The cells of the central anterior lens capsule are considered to be arrested in the $\mathrm{G}_{0-1}$ phase of the cell cycle and hence not actively regenerating, while the cells at the equator retain their mitotic activity. ${ }^{+}$

The proliferation of these arrested cells in culture has been attributed to the tissue culture medium being richer in essential constituents than aqueous humour. ${ }^{5}$ However, ultrastructural studies of human posterior capsule membranes and Elschnig's pearls suggest that they are formed by proliferation of the epithelial cells without any change or enrichment of the aqueous humour. ${ }^{67}$ Other workers have suggested

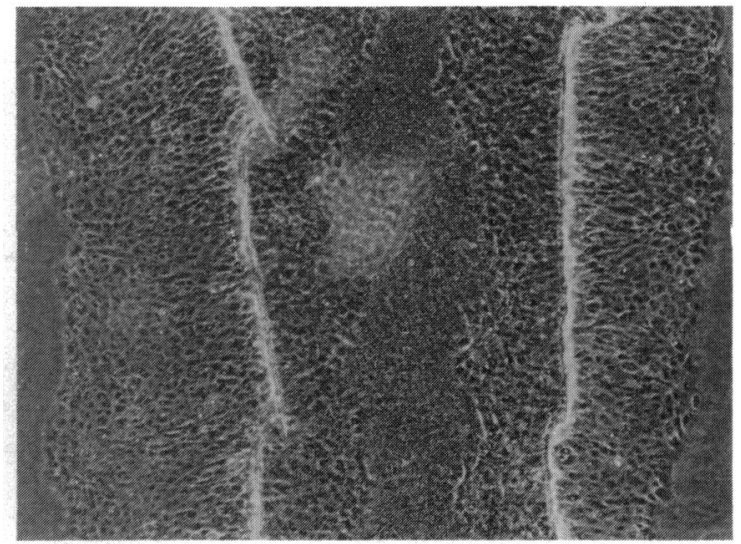

Fig. 3B: Central region of anterior lens capsule.

that a retinal growth factor, uveal epithelial cells, and inflammation may play a role in stimulating anterior capsule cell proliferation. ${ }^{8-11}$

Our culture model excludes the influence of all these possible factors and demonstrates that the primary site of cell proliferation is from the cut edge of the capsule. We believe that release from contact inhibition is a major factor in triggering cellular proliferation, since multilayering of cells is not observed in the initial stage. In our culture cellular proliferation was found to occur across any suitable surface: outwards across the culture substrate and around the anterior edge of the capsule. This has been noted in histopathological studies. "We found no significant difference in growth rate between cells from cataractous and normal lenses. We were limited in our choice of method for determining growth rate by the small amount of available starting material. Thus it was decided to opt for counting the number of cells from the edge of the explant as described in 'Material and methods.' Although this method may not be as precise (the error was estimated at $5 \%$ ) as other cell counting techniques, such as ${ }^{3} \mathrm{H}$-thymidine incorporation or dye staining, these techniques necessitate the termination of culture and are less applicable to explant culture. Counting the number of cells eliminates errors due to variations in cell morphology that occur both with time and between cultures, and any measurement error due to the asymmetry of the outgrowth was reduced by counting cells from each of the sides of the rectangular explant and averaging the results. Contrary to our expectations based on animal studies, ${ }^{5}$ we found no difference in the growth potential between cells of the equator and those of the centre of the anterior capsule. Cytological studies have shown no dissimilarity between these cells. ${ }^{12}$

The finding that after six population doublings in 
primary culture outgrowth ceases, and subsequently the outer epithelial cells become increasingly abnormal, is in agreement with the findings of Tassin $e t$ $a l .{ }^{13}$ In the human, wrinkling of the posterior capsule may be caused by contraction of hyperplastic lens epithelial cells that have acquired myofibroblastic differentiation." Retina derived growth factor has been shown to stimulate differentiation of rat lens epithelial cells, and it has been proposed that neural retina secretes a discrete factor(s) which interacts with epithelial cells as they are displaced into the vitreous compartment from the equator. ${ }^{914}$ In another study on mouse lens epithelial cells the formation of lentoid bodies could be triggered by the addition of a strip of capsule. ${ }^{15}$

The clinical implications of our study are that an anterior capsulotomy provides a stimulus for epithelial cell proliferation. Varying the location of the capsulotomy does not expose cells of greater proliferative potential. Creating a large capsulotomy will not reduce the risk of opacification by decreasing the number of epithelial cells in the eye, as suggested by Green and McDonnell." On the contrary a large capsulotomy, especially if irregular or zig-zag as in the 'can opener' technique, releases a larger area of epithelial cells from contact inhibition. There is thus a theoretical advantage in performing a small capsulotomy or even a 'letter box' capsulotomy as in the endocapsular technique. Conversely the radial distance that proliferating anterior capsule cells have to migrate before occluding the visual axis is decreased with a small capsulotomy. ${ }^{\text {? }}$

In summary, our study has shown that after extracapsular cataract extraction the remaining anterior capsule has a normal pattern of epithelial cells that are firmly adherent to its posterior surface. The edge cells of the cut capsule can proliferate in culture. A significant factor governing their growth is loss of contact inhibition, and it is this loss of contact inhibition that is chiefly responsible for capsule opacification. The role of inflammatory mediators and growth and inhibitory factors in lens epithelial cell proliferation requires further study.
We are grateful for the assistance of Dr A C Thomas, Dr M G Cook, and Mr Gary Thompson for the preparation of the histological slides.

T J C J is an MRC senior research fellow, and funds from the Visual Research Trust are gratefully acknowledged.

\section{References}

1 Jaffe NS. Results of intraocular lens implant surgery. Am J Ophthal 1978; 85: 13-23.

2 Aryon RD, Greisennans JC, Aron JJ. Use of a pulsed neodymium YAG laser (picosecond) to open the posterior capsule in traumatic cataract - a preliminary report. Ophthalmic Surg 1981 12: 496-9.

3 Terry AC, Stark WK, Maumenee AE, Fagadan W. NeodymiumYAG laser for posterior capsulotomy. Am J Ophthalmol 1983; 96: 716-20.

4 Harding CV, Reddan JR, Unakar NJ, Bagchi M. The control of cell division in the ocular lens. Int Rev Cytol 1971; 31: 215-300.

5 McAvoy JW, McDonald J. Proliferation of lens epithelial explants in culture increases with age of donor rat. Curr Eye Res 1984; 3: 1151-3.

6 Kappelhof JP, Vrenson GFJM, de Jong PTVM, Pameyer J, Willikens B. An Ultrastructural study of Elschnig's pearls in the pseudophakic eye. Am J Ophthalmol 1986; 101: 58-69.

7 McDonnell PJ, Zarbin MA, Green WR. Posterior capsule opacification in pseudophakic eyes. Ophthalmology 1983; 90: $15-48$.

8 Odrich MG, Hall SJ, Worgul BV, Trokel SL, Rini FJ. Posterior capsule opacification: experimental analysis. Ophthalmic Res 1985; 17: 75-84.

9 McAvoy JW, Fernon V. Neural retinas promote cell division and fibre differentiation in lens epithelial explants. Curr Eye Res 1984; 3: 827-34.

10 Arruti C, Courtois Y. Morphological changes and growth stimulation of bovine epithelial cells by a retinal extract in vitro. Exp Cell Res 1978; 117: 283-92.

11 Green WR, McDonnell PJ. Opacification of the posterior capsule. Trans Ophthalmol Soc UK 1985; 104: 727-39.

12 Kuwabara T. The maturation of the lens cells: a morphologic study. Exp Eye Res 1975; 20: 427-43.

13 Tassin J, Malaise E, Courtois Y. Human lens cells have an in vitro proliferative capacity inversely proportional to donor age. Exp Cell Res 1979; 123: 388-92.

14 Campbell MT, McAvoy JW. Onset of fibre differentiation in cultured rat lens epithelium under the influence of neural retinaconditioned medium. Exp Eye Res 1984; 39: 83-94.

15 Muggleton-Harris AL, Higbec $N$. Factors modulating membrane and cellular changes with differentiation and morphogenesis of a lentoid structure in vivo.J Embryol Exp Morphol in press.

Accepted for publication 16 September 1986. 